The Astrophysical Journal, 492:540-553, 1998 January 10

(C) 1998. The American Astronomical Society. All rights reserved. Printed in U.S.A.

\title{
A PRELIMINARY STUDY OF THE ORION NEBULA CLUSTER STRUCTURE AND DYNAMICS
}

\author{
LYNNE A. HiLLENBRAND \\ Department of Astronomy, 601 Campbell Hall, University of California, Berkeley, CA 94720; lynne@astron.berkeley.edu \\ AND \\ LEE W. HARTMANN \\ Harvard-Smithsonian Center for Astrophysics, 60 Garden Street, Cambridge, MA 02138; hartmann@cfa.harvard.edu \\ Received 1997 July 7; accepted 1997 August 18
}

\begin{abstract}
We use optical and near-infrared star counts to explore the structure and dynamics of the Orion Nebula Cluster (ONC). This very young $(<1 \mathrm{Myr})$ cluster is not circularly symmetric in projection but is elongated north-south in a manner similar to the molecular gas distribution in the region, suggesting that the stellar system may still reflect the geometry of the protocluster cloud. Azimuthally averaged stellar source counts compare well with simple spherically symmetric, single-mass King cluster models. The model fits suggest that the inner Trapezium region should be regarded as the core of the ONC, not as a distinct entity as sometimes advocated. We estimate that the core radius of the cluster is $0.16-0.21$ $\mathrm{pc}$ and that the central stellar density approaches $2 \times 10^{4}$ stars $^{\mathrm{pc}^{-3}}$. Adopting the stellar velocity dispersion from published proper-motion studies, virial equilibrium would require a total mass within about 2 pc of the Trapezium of $\sim 4500 M_{\odot}$, slightly more than twice the mass of the known stellar population and comparable to the estimated mass in molecular gas projected onto the same region of the sky. If $\gtrsim 20 \%$ of the remaining molecular gas is converted into stars, thus adding to the binding mass, given that the present stellar population alone has a total energy close to zero, the ONC is likely to produce a gravitationally bound cluster. The ONC also exhibits mass segregation, with the most massive (Trapezium) stars clearly concentrated toward the center of the cluster and some evidence for the degree of central concentration to decrease with decreasing mass down to $1-2 M_{\odot}$, as would be expected for general mass segregation. Given the extreme youth of the stars compared with the estimated range of collisional relaxation times, the mass segregation is unlikely to be the result of cluster relaxation. Instead, we suggest that the mass segregation reflects a preference for higher mass stars to form in dense, central cluster regions.
\end{abstract}

Subject headings: celestial mechanics, stellar dynamics open clusters and associations: individual (Orion Nebula Cluster) - stars: evolution

\section{INTRODUCTION}

The Orion Nebula Cluster (ONC) is a star-forming site of extraordinary interest. Relative proximity to the Sun $(d \approx 470 \mathrm{pc})$ combined with low extinction along the line of sight $\left(A_{V} \leq 2.0 \mathrm{mag}\right.$ for the massive Trapezium stars) permits detailed studies of the ONC stellar population over its full range in mass $\left(\sim 0.1-50 M_{\odot}\right.$, with a mean mass $\sim 0.8$ $\left.M_{\odot}\right)$ and age $(<0.1-2 \mathrm{Myr}$, with a mean age of $<1 \mathrm{Myr})$. Hillenbrand (1997) conducted an extensive optical $(I<17.5$ mag) photometric and spectroscopic investigation of ONC members projected within $\sim 2.5 \mathrm{pc}$ of $\theta^{1}$ Ori C, locating 934 representative stars $(\approx 60 \%$ of the total optical sample) on a theoretical H-R diagram and deriving their masses and ages via comparison to pre-main-sequence evolutionary calculations. Evidence was found for gradients with projected cluster radius in both the mass and the age distributions, with the cluster center dominated by the most massive and the youngest stars. However, the optically visible population represents $\leq 50 \%$ of the total stellar census known from infrared surveys.

In this work we combine the optical source counts assembled by Hillenbrand (1997) with infrared source counts from Ali \& DePoy (1995) and McCaughrean \& Stauffer (1994) to explore the structure of the ONC region. In particular, we wish to constrain the relationship between the Trapezium core region (which often has been considered a distinct entity in the past; see Herbig \& Terndrup 1986) and the overall ONC. We also wish to understand the origin of the apparent radial segregation in stellar mass and stellar age in the ONC, as well as to speculate on the future dynamical evolution of the cluster. We are motivated by the fact that the high density of star formation and the large number of stars present in the ONC suggest it may be a proto-open cluster - unlike the young stellar populations in the closer, but significantly sparser, Taurus, Ophiuchus, Lupus, and Chamaeleon star-forming regions. It is likely that stellar populations formed in Taurus-like clouds are bound only by the mass of the molecular gas with which they are currently associated, and so only for the $\leq 10 \mathrm{Myr}$ lifetime of the molecular component. In contrast, the $\leq 1$ Myr old ONC has already removed most of the gas near its center $(r \leq 0.3 \mathrm{pc})$ via dissociation and ionization by the massive stars. That its central density is approximately 2 orders of magnitude above that typical of older Galactic open clusters suggests that the ONC could evolve into a lower-density cluster (such as the Pleiades) in a few tens of Myr. We may thus ask whether some portion of this dense group of stars will produce an initially bound open cluster even after all of the gas has been removed from the system? Furthermore, we may ask whether the ONC might contribute significantly to the field star population through cluster evaporation over many hundreds of Myr and evolve to a remnant open cluster (such as the Hyades). If so, the $\mathrm{ONC}$ and other regions like it can be thought of as impor- 
tant starting points for investigation of the process of star formation appropriate to both Galactic open cluster and field star populations.

Determining the structure and dynamics of the ONC and its central, dense Trapezium region is difficult for several reasons. First, the bright, nonuniform nebulous background and the extreme crowding hinder both the initial detection of stellar sources and the derivation of good photometry for them. As a result, surveys employing different techniques, at different wavelengths of observation, with different spatial resolution, and over varying survey areas must be pieced together to provide a comprehensive picture of star formation in the ONC region. Second, spatially variable extinction is troublesome because although the line-of-sight extinction derived for many of the optical stars on the near side of the cluster is relatively low, high molecular column density behind the cluster corresponding to $A_{V}=50-100$ mag of extinction (Bergin 1996; Castets et al. 1990) introduces the difficulty of having to understand the spatial overlap between the optical/infrared cluster and the highdensity background molecular cloud. This overlap is not at all clear, at present. Third, this background gas constitutes a significant amount of mass. Its spatial distribution on the sky suggests that this gas contributes significantly to the total gravitational potential and that additional star formation will occur in the next Myr or so, increasing the mass in stars.

Given the above, it is difficult to assess the uncertainties and possible incompleteness in our knowledge of the total ONC stellar population. Nevertheless, as much recent progress has been made in improving the stellar census, it is worth making a preliminary investigation of ONC structure and dynamics. In $\S 2$ we describe the current ONC census data. In $\S 3$ we present stellar surface density distributions that allow us to compare the optically visible and invisible clusters and to conclude that they have the same twodimensional structure. In $\S 4$ we review literature information on the cluster velocity dispersion, and in $\S 5$ we describe fits of the ONC data to standard lowered isothermal sphere cluster models. In $\S 6$ we address the possibilities of cluster relaxation, mass segregation, and boundedness.

\section{THE CURRENT ONC STELLAR CENSUS}

In this section we review our knowledge of the optically visible and the optically invisible (embedded) stellar populations in the ONC region. Deep imaging within the innermost few parsecs of the cluster over the past several years has added considerably to the stellar census. We find that the current database appears representative of the spatial distribution of all cluster stars and that it is not grossly incomplete.

As technology has improved with time, so have the stellar census counts and stellar density estimates in the Trapezium/ONC region. Trumpler (1931) noted the existence of the faint but dense stellar grouping associated with the bright Trapezium stars and first called it the Trapezium cluster. Baade \& Minkowski (1937) quantified the extent of the stellar population, finding 80 stars in a $3^{\prime}$ diameter and 130 stars in a $4^{\prime} .6$ diameter, which they took as the cluster size. The stellar surface density computed from these early numbers is about $600 \mathrm{stars} \mathrm{pc}^{-2}$ inside a radius of $0.2 \mathrm{pc}$ and about 420 stars $\mathrm{pc}^{-2}$ inside a radius of $0.3 \mathrm{pc}$, which approximates the expected $1 / r$ relationship for surface density in an isothermal cluster.
For several decades hence, the stellar population in the inner ONC was not reexamined (although much work was done in the outer regions; see Parenago 1954; Walker 1969; Andrews 1981). Then Herbig \& Terndrup (1986) used red CCD imaging to find 142 stars in a region $9.1 \mathrm{arcmin}^{2}$ in size $(0.23 \mathrm{pc}$ in equivalent radius) and computed a stellar volume density of 2200 stars $^{-3} \mathrm{pc}^{-3}$, making the Trapezium cluster the densest nearby star-forming region known. ${ }^{1}$ Herbig \& Terndrup also compared their optical with existing infrared magnitude distributions, to conclude that the high stellar density was local to the Trapezium cluster itself and not a product of contamination by stars located behind the cluster in the molecular cloud. Jones \& Walker (1988) catalogued 1053 stars from red photographic plate material over a $700 \mathrm{arcmin}^{2}$ area. Prosser et al. (1994) used HST imaging capabilities at the $I$ band to find 319 stars (180 new) in $12 \mathrm{arcmin}^{2}$. Further HST imaging by O'Dell \& Wong (1996) produced $\approx 120$ new sources in the inner $50 \mathrm{arcmin}^{2}$. Hillenbrand (1997) added 350 previously uncataloged optical stars over a $900 \mathrm{arcmin}^{2}$ area. In the infrared, McCaughrean (1987) used $K$-band imaging to find 500 stars within a 25 arcmin $^{2}$ area, and McCaughrean \& Stauffer (1994) used high-resolution near-infrared imaging to find 123 sources over $1.9 \operatorname{arcmin}^{2}$. Ali \& DePoy $(1995)^{2}$ cataloged 3548 stars over $1472 \mathrm{arcmin}^{2}$ in the largest published survey to date.

The deeper among these surveys have led to revised estimates of the Trapezium cluster central stellar density. Using Monte Carlo simulations to correct for the stars appearing at smaller projected radii than their true central distances, McCaughrean \& Stauffer (1994) and Stauffer et al. (1994) computed a peak volume density in the Trapezium of $\approx 5 \times 10^{4}$ stars $\mathrm{pc}^{-3}$ inside of $0.1 \mathrm{pc}$. McCaughrean \& Stauffer also estimated that the stellar volume density external to this region appears to fall off as $1 / r^{2}$ over several tenths of a parsec.

In the following analysis we consider two source samples that extend over $\sim 2.5 \mathrm{pc}$ in radius from $\theta^{1}$ Ori C. Our "optical" sample consists of 1578 sources taken from the $I$-band surveys described above and is extensively discussed in Hillenbrand (1997). In the outer cluster regions where the nebular background is not so bright, the photometric database is reasonably complete down to $I \sim 17.5 \mathrm{mag}$. In the inner regions where deeper HST surveys have been conducted, completeness extends to $I \sim 19 \mathrm{mag}$. However, at least for the ground-based data, the completeness for point source detection is approximately $1.5 \mathrm{mag}$ deeper than the completeness for accurate photometry (based on experimentation with the addition of artificial stars to the images). Thus, the variation in survey depth with cluster radius is probably less severe than one might initially conclude. We

\footnotetext{
${ }^{1}$ An updated stellar census (see below) confirms that the Trapezium region is the densest of nearby star-forming regions. However, recent work on the giant H II regions NGC 3603 in the Galaxy, R136a in the Large Magellanic Cloud, and NGC 604 in M33-including the resolution of apparently single supermassive objects into dense clusters of massive stars-indicates these regions to be more dense than the Trapezium region by factors of several, if it can be assumed that these more distant high-mass clusters have complementary low-mass components with mass spectra like that of the ONC.

${ }^{2}$ Approximately $10 \%$ of the Ali \& DePoy sources turn out to be duplicates, that is, multiple positions quoted in their Table 2 that fall within 0.5 of one another despite the 1".5 platescale of their images. In all of these cases, however, the photometric measurements are (nearly) identical, and we could eliminate duplications.
} 
augment the "optical" sample with an "infrared" sample of 1997 sources taken from the $K$-band surveys described above. As with the optical data, the infrared data are about $1.5 \mathrm{mag}$ deeper in the inner regions than in the outer regions, with the larger Ali \& DePoy (1995) survey reportedly complete to $K=14.5$ and the McCaughrean \& Stauffer (1994) survey over the inner $0.2 \mathrm{pc}$ complete to $K=16$. The "optical" and "infrared" data sets are mutually exclusive.

Out to a radius of $\sim 2.5 \mathrm{pc}$ from $\theta^{1}$ Ori $\mathrm{C}$, the current cluster census numbers $\sim 3500$ stars in total. Have all the stars formed as part of the ONC now been detected? The factor of 2 increase in source counts from the optical to the infrared surveys suggest a substantial population of sources with large values of extinction. This could mean that, in fact, much of the stellar population does remain to be uncovered; alternatively, the infrared surveys may be already beginning to probe the stellar distribution that is located in the background cloud and that is not part of the dynamical cluster. We argue that summing together existing optical and infrared databases provides a representative, relatively uncontaminated, and perhaps near-complete picture of the $\mathrm{ONC}$, for the following reasons:

1. Deep surveys in the inner cluster regions suggest completeness.-Large nebular background and extreme stellar crowding dominate the problem of source detection in the inner cluster. However, it is within the central region of the ONC that the stellar population is sampled to the faintest magnitudes and at the highest spatial resolution. In this innermost region, the McCaughrean \& Stauffer (1994) infrared survey found fainter, presumably more extincted, stars than did the Prosser et al. (1994) and the O'Dell \& Wong (1996) optical surveys. However, the extra infrared sources increased the census determined from optical data over this region by only about $30 \%$. Furthermore, the sharp decline in the $K$-band magnitude function beyond $K=13$ mag found by McCaughrean et al. (1995), based on a $0.7 \times 0.7$ pc survey complete to $K \sim 17 \mathrm{mag}$, suggests the faint limit of the ONC population may have been detected.

2. Survey completeness in the outer cluster is only slightly less than that in the inner cluster.-Although the McCaughrean \& Stauffer (1994) and McCaughrean et al. (1995) surveys extend several magnitudes fainter than the larger Ali \& DePoy (1995) survey, the sharp turnover in the $K$-band magnitude function below $K=13 \mathrm{mag}$, with less than $1 \%$ of the McCaughrean \& Stauffer sample fainter than $K=15$ mag, suggests that the less deep Ali \& DePoy survey, complete to $K=14.5 \mathrm{mag}$, is in fact not far from being fully complete.

3. There is no evidence for significant numbers of heavily extincted stars.-While the large column density in the molecular cloud behind the ONC is certainly effective in suppressing contamination by any background population, it is also capable of concealing some genuine cluster stars. If a large number of cluster stars are in fact hidden within the $A_{V} \approx 50-100$ mag background molecular cloud, this could compromise our goal of understanding the cluster dynamics since the cluster potential could then be dominated by a gaseous-not its stellar-component. The results of Hillenbrand (1997) suggest that the mean extinction for the optically visible ONC population is $A_{V} \lesssim 2.5 \mathrm{mag}$, with few optical stars having $A_{V} \gtrsim 5$ mag. Extinction estimates for the infrared sample can be estimated using $J H K$ photometry and the dereddening technique described by Meyer
(1996), assuming the infrared population has the same distribution of stellar ages as the optical population (see Hillenbrand et al. 1998 for a detailed description). The mean extinction so derived for the infrared sample is about a factor of 3 higher than the mean extinction of the optical sample, with a relatively small fraction of the infrared population suffering large extinction $\left(A_{V} \gtrsim 15 \mathrm{mag}\right)$. However, as the infrared dereddening technique requires stars to have photometry at the $J, H$, and $K$ bands, the sources that are faintest at $K$ generally are not detected at shorter wavelengths in the current surveys and thus do not yet have extinction estimates. Nevertheless, there is no indication at present of rising source counts toward higher extinction values (the extinction distribution for the total optical + infrared sample is flat from $A_{V}=0-8 \mathrm{mag}$ and then falls off with increasing extinction to $A_{V} \sim 15 \mathrm{mag}$ ), and so we suspect that we are not seeing a substantial number of stars located in the background molecular cloud within the current database. Combined with the turnover in the $K$-magnitude function, these estimates render it unlikely that such a sample of heavily extincted stars even exists, although deeper $J H K L$ surveys would be required to confirm this assertion.

4. The projected spatial distributions of the optical and the infrared sources are the same.--If the gas and dust in the vicinity hides much of the cluster rather than simply providing a background screen, one might expect to see differences in the spatial distribution of the infrared and optical sources. To explore this, we binned the star counts for the optical-only and the infrared-only samples into 10 ".8 pixels and then smoothed with a two-dimensional symmetric Gaussian profile of $\sigma=3$ pixels to produce 32".4 pixels. Figure 1 (top and bottom panels) shows the spatial distributions of these two smoothed samples, along with contours drawn at factors of 2 in surface density. The panels display very similar spatial structure. To quantify any differences between the distributions, we divided the optical-only image by the total image (optical + infrared; see Fig. 3) and show the results in Figure 2, again with contours at factor of 2 spacing in surface density. The divided image is remarkably uniform (except around the border, as a result of the infrared survey area slightly exceeding the optical survey area; see Fig. 1) and gives no hint that the spatial distribution of the optical sample differs in any general way from that of the total sample. Extinction features are apparent, most noticeably the region of low counts in Figure 2 running diagonally southeast-northwest across the image just north of the central Trapezium region. This "streak" of low optical source counts can be seen directly in the unbinned optical data and most likely indicates a band of higher extinction in this region. But even this most striking feature does not affect the overall similarity of the structure between the optical and the optical + infrared source count distributions. Other strong minima in this divided image are in regions of very low optical source counts and therefore are not statistically significant. In the innermost regions, the optical source counts follow the optical + infrared sample remarkably well. There is a maximum in the relative number of optical source counts at the very center ( $\sim 70 \%$ of the total), where $H S T$ has provided the deepest optical data, as discussed above.

The above results are consistent with a picture in which a large fraction of the stars in the ONC have been cataloged in existing photometric databases. How complete are these 

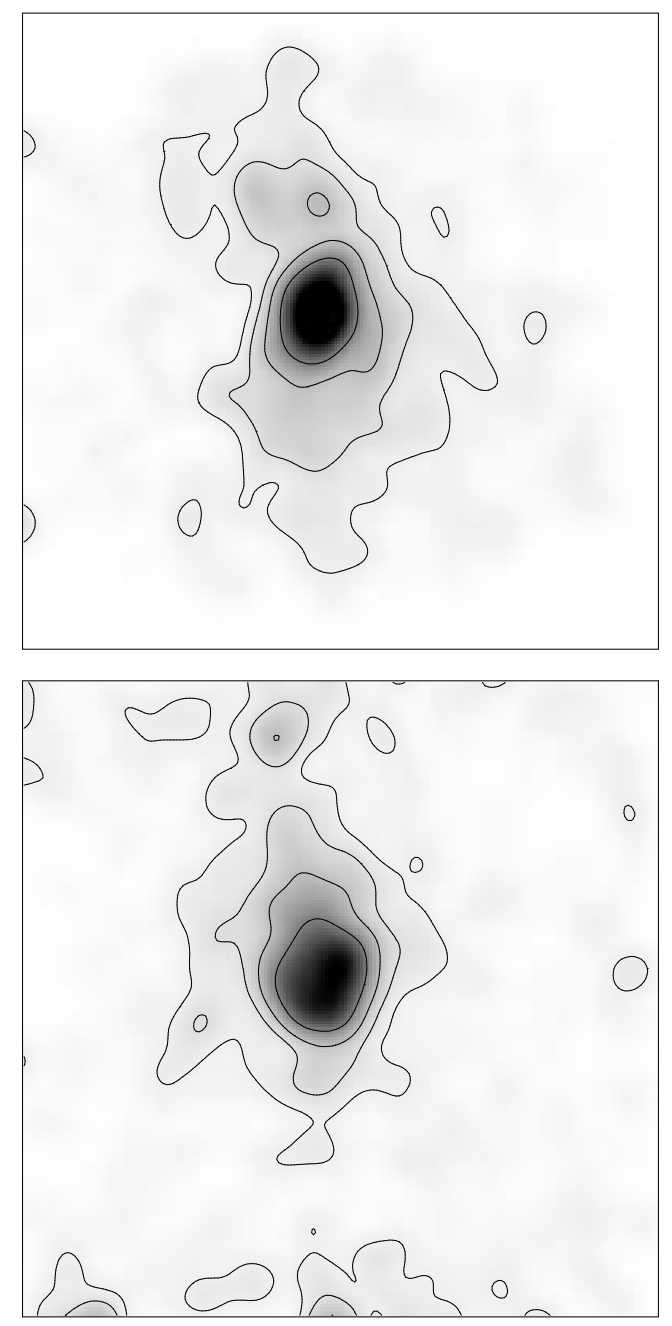

FIG. 1.-Surface density distribution of stars for the optical-only sample (top panel) and the infrared-only sample (bottom panel), selected and smoothed with a Gaussian profile of width $\sigma \approx 32^{\prime \prime}$, as described in the text. Contours are shown every factor of 2 increase in surface density. The area in each panel is $36 \times 36 \mathrm{arcmin}^{2}$ centered at R.A. $=83^{\circ} .8$, decl. $=$ $-5.4\left(\approx 1^{\prime}\right.$ west of $\theta^{1}$ Ori $\left.C\right)$, with north up and east to the left.

databases in terms of stellar masses, stellar ages, and extinction? The optically visible stellar population includes stellar masses extending down to and possibly below the hydrogen-burning mass limit. The mass spectrum is roughly consistent with (although different in detail from) the Miller \& Scalo (1979) function, which has a median mass of 0.3 $M_{\odot}$, corresponding to a spectral type of M2 for an age $\lesssim 10^{6}$ yr. A "typical" ONC star has a slightly later spectral type of M3-M4, and with a "typical" $A_{V} \sim 1.3$ has $V \sim 17.5, K \sim 12$ at the distance of Orion for the $10^{6} \mathrm{yr}$ isochrone. So outside the region where the deepest imaging surveys have been conducted (i.e., at $r>5^{\prime}$ ), if the optical sample becomes incomplete below $V \sim 19, \quad I \sim 17.5$ (Hillenbrand 1997) and if the infrared sample becomes incomplete below $K \sim 14.5$ (Ali \& DePoy 1995), but the infrared sample roughly doubles the total number of stars, then the infrared sample must include many more stars that have essentially the same spatial distribution and mass distribution but that are located behind larger values of extinction. The properties of the embedded population are discussed in more detail in Hillenbrand et al. (1998). We conclude that the factor of 2 incompleteness in the optical sample is the result of the extinction effects, but we remind the reader of our arguments above on the nearcompleteness of the optical + infrared sample.

With confidence that the existing optical + infrared point source surveys in the ONC are representative and, furthermore, not vastly incomplete, we can begin to investigate the cluster structure and dynamics.

\section{SURFACE DENSITY DISTRIBUTIONS}

We begin an initial reconnaissance of cluster structure by fitting elliptical isocolumn density contours to the observed star counts, shown in Figure 3. As described above, the star counts were binned into $10^{\prime \prime} 8$ pixels and then smoothed with a two-dimensional symmetric Gaussian profile of width $\sigma=3$ pixels. This smoothing aided the subsequent isodensity analysis, especially on large scales. We then used the ellipse isophote-fitting program of the STSDAS analysis package to search for centroids and elliptical isodensity contours, using the mean area integration mode. During the fitting procedure we assumed initially circular isophotes and then allowed the center and the ellipticity to vary. We found that ellipses with semimajor axes in the range $\sim 50^{\prime \prime}-$ $200^{\prime \prime}$ generally gave similar results. Table 1 reports sample results for both the optical and optical + infrared samples. The inner three isophotal contours in both samples yield reasonably consistent results for the cluster centroid and ellipticity. Fits for larger semimajor axes did not yield statistically significant results, as indicated by the error estimates; this is not surprising, since on the largest scales the isodensity structure appears much more like a filament than an ellipsoidal distribution.

The average centroid positions suggest a center near the right ascension of $\theta^{1}$ Ori $\mathrm{C}$ (the most massive star in the ONC) but perhaps up to $\sim 25^{\prime \prime}$ north. The centroid fits on the smallest scale lie closer, within $10^{\prime \prime}$. As discussed further in the following section, it may not be possible at this point to determine the "center" of the cluster to much better than $10^{\prime \prime}-20^{\prime \prime}$.

The ellipse fitting on scales $\lesssim 200^{\prime \prime}$ give a consistent estimate of the ellipticity of $\epsilon=0.30 \pm 0.02$, with a position angle nearly north-south, $\theta=-14^{\circ} \pm 6^{\circ}$. The results are similar for the optical-only sample. It is suggestive that both the large-scale distribution of infrared sources (Ali \& DePoy 1995) and the filamentary molecular gas in the region (Castets et al. 1990; Bergin 1996) are both extended north-south; the inner cluster structure may reflect this tendency as well.

The modest ellipticities found by this procedure, along with the uncertainties in the stellar census, suggest that for simplicity an initial exploration of cluster structure can be made from stellar surface densities binned in azimuthal averages. Figure $4 a$ shows the azimuthally averaged number of optical sources per unit area in logarithmic distance bins for two choices of centroid; the "fit" is centered on the optical-only mean position given in Table 1, while the other distribution assumes that $\theta^{1}$ Ori $\mathrm{C}$ defines the cluster center. Figure $4 a$ indicates that there is little difference in source counts adopting either center; centering on $\theta^{1}$ Ori $\mathrm{C}$ provides a slightly more peaked central density, so we adopt it in what follows, but the precise choice of center does not affect our results. Figure $4 b$ shows a comparison between the optical-only sample and the optical + infrared sample. As discussed above, including the infrared sources roughly doubles the total number of objects but does not 


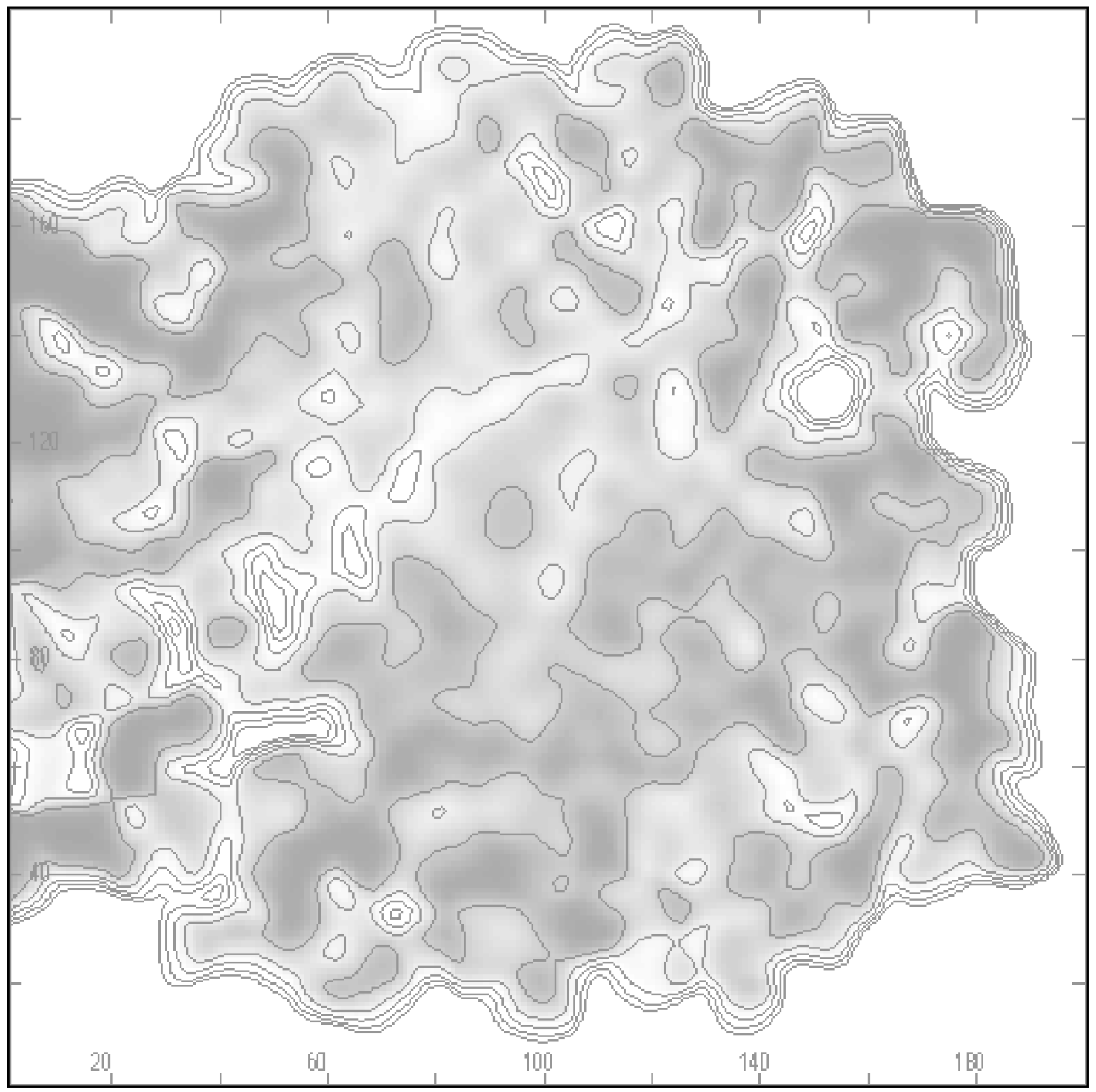

Fig. 2.- Ratio of optical-only surface density to the optical + infrared surface density. The size and orientation of the image are the same as in Fig. 1 . Light shading indicates low values in the divided image, i.e., regions of proportionally larger contribution from the infrared sample relative to the optical sample. Contours denote factor of 2 differences in surface densities. For reference, the contour around the central (Trapezium core) region corresponds to a ratio of 0.64 , while the contours around the two brighter regions to the immediate northwest and southwest of the central region have a ratio of 0.32 . The lack of structure in this divided image provides the basis for our conclusion that the optically visible and the infrared clusters are not structurally different from one another.

TABLE 1

ONC Ellipse Fitting

\begin{tabular}{|c|c|c|c|c|}
\hline $\begin{array}{l}\text { Semimajor Axis } \\
\quad(\operatorname{arcsec})\end{array}$ & $\begin{array}{c}\alpha(2000)-\alpha\left(\theta^{1} \text { Ori } \mathrm{C}\right) \\
(\operatorname{arcsec})\end{array}$ & $\begin{array}{c}\delta(2000)-\delta\left(\theta^{1} \text { Ori } \mathrm{C}\right) \\
(\operatorname{arcsec})\end{array}$ & Eccentricity & $\begin{array}{l}\text { P.A. } \\
\text { (deg) }\end{array}$ \\
\hline \multicolumn{5}{|c|}{ Optical Only, 32" Smoothing } \\
\hline $54 \ldots$ & $-3.6 \pm 0.01$ & $10.7 \pm 0.01$ & $0.29 \pm 0.004$ & $-13.2 \pm 0.4$ \\
\hline $108 \ldots \ldots \ldots \ldots \ldots \ldots$ & $-12.4 \pm 0.09$ & $24.1 \pm 0.13$ & $0.32 \pm 0.018$ & $-7.5 \pm 1.9$ \\
\hline & $-5.6 \pm 0.38$ & $9.5 \pm 0.51$ & $0.30 \pm 0.036$ & $-13.9 \pm 4.1$ \\
\hline $532 \ldots \ldots \ldots \ldots \ldots$ & $11.1 \pm 0.75$ & $10.5 \pm 1.39$ & $0.54 \pm 0.032$ & $3.2 \pm 2.6$ \\
\hline Mean, $54-216 \ldots \ldots$ & $-7.2 \pm 4.6$ & $14.8 \pm 8.1$ & $0.30 \pm 0.02$ & $-11.6 \pm 3.6$ \\
\hline \multicolumn{5}{|c|}{ Optical + Infrared, $32^{\prime \prime}$ Smoothing } \\
\hline $54 \ldots \ldots \ldots \ldots \ldots$ & $2.7 \pm 0.02$ & $9.0 \pm 0.03$ & $0.33 \pm 0.01$ & $-18.2 \pm 0.7$ \\
\hline $108 \ldots \ldots \ldots \ldots \ldots$ & $-3.1 \pm 0.07$ & $19.4 \pm 0.10$ & $0.34 \pm 0.01$ & $-17.3 \pm 1.3$ \\
\hline $216 \ldots \ldots \ldots \ldots \ldots$ & $-4.1 \pm 0.18$ & $34.5 \pm 0.24$ & $0.28 \pm 0.02$ & $-6.8 \pm 2.2$ \\
\hline $532 \ldots \ldots \ldots \ldots \ldots \ldots$ & $-7.0 \pm 0.58$ & $67.9 \pm 0.95$ & $0.49 \pm 0.025$ & $7.1 \pm 2.2$ \\
\hline Mean, $54-216 \ldots . .$. & $-1.5 \pm 3.7$ & $21.0 \pm 12.8$ & $0.31 \pm 0.03$ & $-14.1 \pm 6.3$ \\
\hline
\end{tabular}




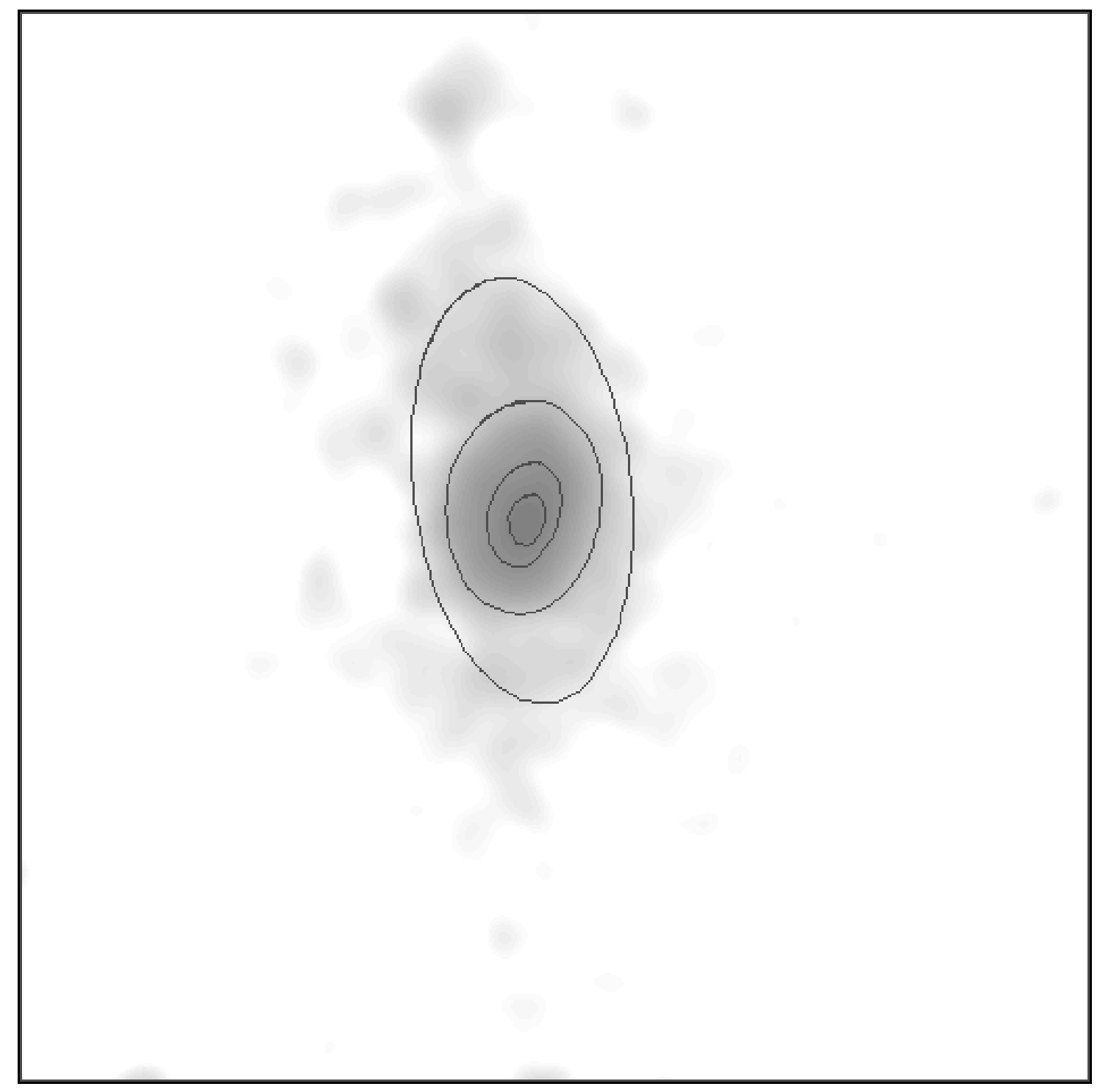

Fig. 3.-Result of ellipse fitting to the projected surface density distribution for the optical + infrared sample (gray scale), as discussed in the text. The contours are for ellipses with semimajor axes of 54", 108", 216", and 532" (see Table 1). The size and orientation of the image are the same as in Fig. 1.

change the overall appearance of the source counts versus radius. Finally, the crosses in Figure $4 b$ show the average densities from the ellipse fitting, plotted as a function of semimajor axis instead of radius. The good agreement with the azimuthal average suggests that we are not deriving a strongly biased view of the source count densities by averaging over the possibly flattened cluster structure.

\section{VELOCITY INFORMATION}

In order to interpret the observed surface density distribution in terms of a physical cluster model, we need some estimate of the space velocities of cluster members and the derived velocity dispersion. Orion is close enough to us that both proper-motion and radial velocity measurements are available, although the size of the sample for which threedimensional space motions are known is small (only a few bright stars). If the individual stellar velocities indicate random motion, then it is likely that the current orbits of the stars are not too different from their initial orbits. On the other hand, if ordered motions (i.e., velocity anisotropies) can be detected, then the cluster may have evolved beyond its initial state. Comparison of the mean stellar velocity dispersion and the velocity dispersion of the gas can help determine whether the cluster dynamics are still related to the cloud dynamics or if they are totally independent of the cloud potential.

Strongly differing views on the ONC dynamics have been proposed in the past based on different data sets. The dispersion in the three-dimensional space velocities of cluster members, with some stars moving toward and some away from the cluster center, and no apparent trends in the radial or tangential motions with projected cluster radius, led Walker (1983) to argue that star formation has occurred all throughout the ONC and that there is no bulk motion either inward or outward of the stellar population. Thus, the stars can be thought of as being located on their initial orbits. However, although Jones \& Walker (1988) found a velocity distribution that is basically isotropic, with a onedimensional dispersion of $2.34 \pm 0.09 \mathrm{~km} \mathrm{~s}^{-1}$, they interpreted a very slight trend of decreasing tangential velocity dispersion and increasing radial velocity dispersion-both 

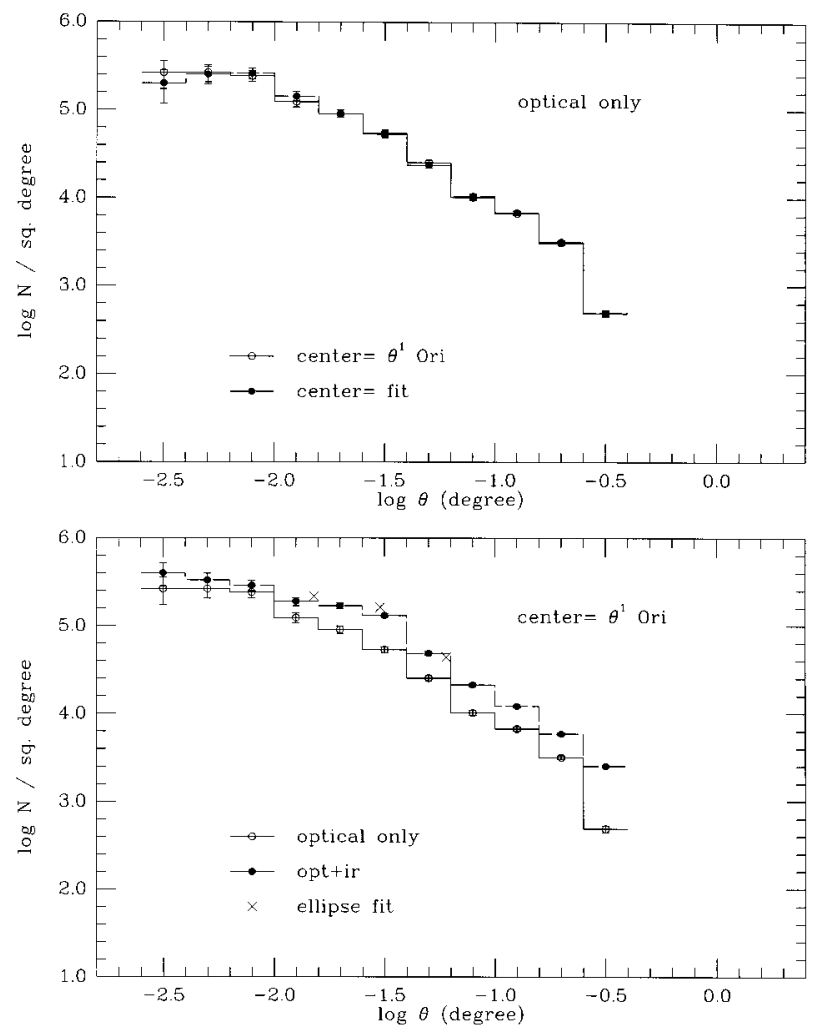

FIG. 4.-ONC surface density distributions. In the upper panel, source counts are shown for the optical-only sample in azimuthally averaged logarithmic bins centered on $\theta^{1}$ Ori C (open circles) and on the position of a centroid fit to the data (filled circles); see text and Table 2. There is essentially no difference in the projected surface densities for the different centers except in the innermost bins. In the lower panel, surface densities for the optical and the optical + infrared samples are compared. The overall trends are similar for these two samples, but there is a difference of about a factor of 2 in the ordinate. Crosses indicate the isodensity measurements from ellipse fitting to the optical + infrared sample (Fig. 3), with the abscissa in this case the ellipse semimajor axis rather than radius. Note in both the upper and lower panels that the error bars refer only to counting statistics and do not take any other source of error into account. The last data bin is probably unreliable because of limits in the survey area.

with increasing radius - as evidence for expansion of the cluster. $^{3}$

In addition to the trend with cluster radius, there is also a suggested trend in velocity dispersion with stellar mass. Both the radial velocity (Walker 1983; Johnson 1965) and proper-motion (Jones \& Walker 1988; van Altena et al. 1988) data show the same increase in dispersion toward later type and fainter (i.e., lower mass) stars. For the radial velocities, the magnitude of the effect is a factor of $\sim 3$ (see Walker 1983) over the spectral type range B-K. For the proper motions, while van Altena et al. (1988) found the one-dimensional velocity dispersion of the $\approx 50$ brightest members $(I<11 \mathrm{mag})$ to be $1.49 \pm 0.2 \mathrm{~km} \mathrm{~s}^{-1}$, Jones \& Walker found a larger velocity dispersion of $2.34 \pm 0.09 \mathrm{~km}$ $\mathrm{s}^{-1}$ for their total sample of $\sim 900$ stars brighter than $I \leq 16 \mathrm{mag}$. Jones \& Walker confirmed the van Altena results by presenting evidence for an increase by about $50 \%$ in the velocity dispersion with apparent magnitude from

\footnotetext{
${ }^{3}$ In contrast, Fallon, Gerola, \& Sofia (1977) derived a radial contraction term of $4.6 \mathrm{~km} \mathrm{~s}^{-1} \mathrm{pc}^{-1}\left(16 \mathrm{~km} \mathrm{~s}^{-1}\right.$ at their outer radius of $\left.3.5 \mathrm{pc}\right)$ from proper motions of 120 stars; the validity of this result may be called into question, however, based on the poor agreement between the proper motions of these authors and those of several subsequent investigators.
}

$I=9-13$ but a flat relationship between $I=13-16$. Because mass estimates exist for 738 of the stars for which Jones \& Walker derived proper motions (665 of which are designated as cluster members), we can investigate this suggested trend directly. Dividing the stellar mass range into logarithmic intervals of 0.5 dex and recalculating the velocity dispersion within each mass bin, we confirm the existence of a slight trend of increasing velocity dispersion toward lower masses. We find $\langle\sigma\rangle=2.81 \mathrm{~km} \mathrm{~s}^{-1}$ for $0.1<M / M_{\odot}<0.3$ stars and $\langle\sigma\rangle=2.24 \mathrm{~km} \mathrm{~s}^{-1}$ for $1<M / M_{\odot}<3$ stars. However, for a cluster in energy equipartition, the massive stars should impart energy to the less massive stars such that the two components follow

$$
\frac{\sigma_{1}}{\sigma_{2}}=\sqrt{\frac{m_{2}}{m_{1}}} .
$$

Such a strong mass dependence of the velocity dispersion is not seen in the ONC, with the data showing a factor of 10 change in mass producing only about a $25 \%$ change in velocity dispersion. This suggests that the ONC can be adequately described by a single-velocity-dispersion model.

There is little evidence for velocity anisotropy in the proper-motion data, with the ratio of the radial velocity dispersion to the tangential velocity dispersion different from unity only by $2 \%$ in the Jones \& Walker study. For a characteristically brighter sample of stars, van Altena et al. found a ratio of radial-to-tangential velocity dispersion of 1.34 but interpreted it as being consistent with an isotropic velocity distribution.

\section{CLUSTER MODELS}

In an exploratory attempt to understand the ONC stellar distribution in physical terms, we compare the observed source counts with the typical models used for cluster structure, i.e., King or lowered isothermal sphere models (see Binney \& Tremaine 1987). With these isotropic models one assumes a distribution function in energy $\left(E=1 / 2 v^{2}+\Phi\right)$ only, instead of a more general distribution function that also includes angular momentum $(J=r v)$. One then integrates the distribution function, $f(E)$, over all velocities and solves Poisson's equation to determine a radial density profile that is compared with the observations.

The applicability of King models to the ONC cluster is uncertain for the following reasons: (1) the possibility that the cluster is not yet close enough to dynamical equilibrium for these models to apply (e.g., the cluster is not spherical, and the molecular gas may play an important role in establishing the gravitational potential); (2) the implicit assumption in the models that the stellar distribution can be treated in a statistical manner (so that as the system evolves because of two-body encounters, the change in velocity produced by any single encounter can be considered as small), strictly true only for large-number samples; ${ }^{4}$ (3) the probable lack of a truly isotropic velocity distribution (in which case the Michie-King distribution function in energy and angular momentum is more appropriate) given that the

\footnotetext{
${ }^{4}$ Retterer (1979) found that the differences between the solutions of the Fokker-Planck equation (on which the King models are based) and the solutions of the Kolmogorov-Feller equation (which is more appropriate for finite numbers of stars) go approximately as $1 / \ln N$, independent of velocity. Thus, the application of dynamical equilibrium models to the current sample of only several thousand stars is valid, with the primary effect being on the tail of the velocity distribution.
} 
cluster is not yet old enough to have undergone collisional relaxation; ${ }^{5}$ and (4) the large range in mass $\left(0.1-50 M_{\odot}\right)$ among the cluster stars, which implies that a separate distribution function should be considered for each mass component. Nevertheless, we feel that comparison with simple physical models is justified for exploratory purposes. The $\mathrm{ONC}$ is the only nearby young cluster for which the stellar population is large enough (and sufficiently well studied) to begin attempting a comparison, and the King models involve a minimum number of parameters. The models enable us to translate observed surface densities into physical volume densities for studying the cluster structure. In the remainder of this section we describe the fitting procedure for matching King models to the ONC surface density distribution and the resulting cluster physical parameters.

\subsection{Fitting Procedure}

A cluster model is described by its central potential $W_{0}=$ $\Psi(0) / \sigma^{2}$ and is scaled to the observations via the core radius $\left[r_{0}\right.$, defined as the projected radius at which the surface density is approximately one-half of its central value, $\left.\Sigma\left(R_{0}\right)=0.5 \Sigma(0)\right]$ and either the central mass density $\left(\rho_{0}\right)$ or the one-dimensional velocity dispersion $(\sigma)$. Specifying the cluster concentration (the logarithmic ratio of the core radius, $r_{0}$, to the truncation radius, $r_{t}$ ) is equivalent to specifying the central potential. We found agreement using both the optical-only and the combined optical + infrared source counts with the simple King cluster models. Comparison with the Hubble model (which is essentially equivalent to the King model except that it does not take truncation into account) shows that the effect of an outer truncation radius is negligible, especially so since the outermost radial bins are affected by the limited survey areas. The core radii derived for the optical-only and the optical + infrared samples are similar (although slightly smaller values are found for the optical-only sample), and so for statistical purposes we focus our efforts to derive the best model fits on the total optical + infrared sample. The models are all for the same cluster concentration since the results are not very sensitive to the truncation radius.

In Figure 5 we compare the King models with the optical + infrared source counts summed in logarithmic spatial bins. Including only those bins from $\log \theta=-2.5$ to $\log \theta=-0.7$, the $\chi^{2}$ values show a broad minimum for core radii in the range of $90^{\prime \prime}-65^{\prime \prime}$, or about 0.21 to about 0.16 pc. These results are consistent with the analysis of McCaughrean \& Stauffer, who tried to fit a King model with a core radius of $30^{\prime \prime}$ to their data and found that the model was too sharply peaked compared with their source counts. For the 10 data bins considered, the best values of $\chi^{2}$ were around $40-45$. Although these high values nominally indicate a poor fit, it was assumed during the fitting procedure that the only source of error is counting statistics. This is highly unlikely, given the problems of nonuniform sampling, variable extinction, departures from spherical symmetry, etc. If the errors in source counts in the spatial range around $70^{\prime \prime}-180^{\prime \prime}$ were more like $3 \%-4 \%$ rather than the $\sim 1 \%-2 \%$ derived from counting statistics, the $\chi^{2}$ values

${ }^{5}$ All available evidence (see $\S 4$ ) suggests, however, that there is no anisotropy, and even if so, such an effect would influence only the highvelocity tail of the distribution.

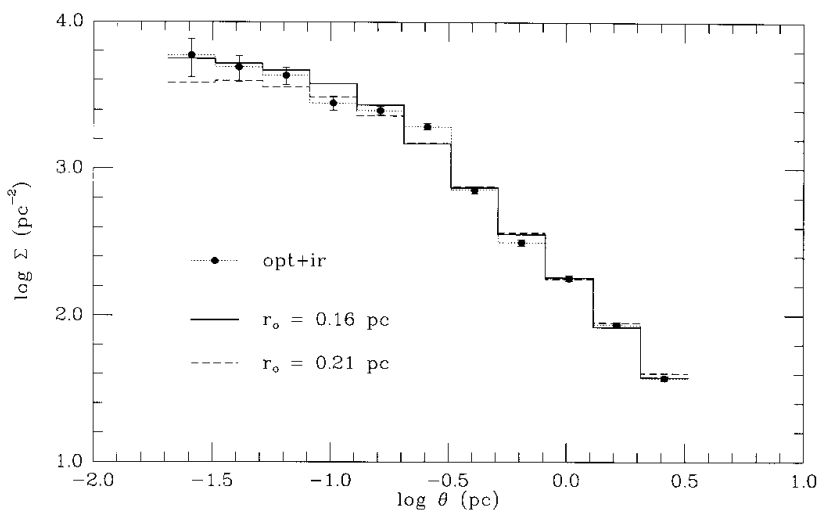

FIG. 5.-Comparison of cluster models (solid and dashed lines) with observed source counts (dotted line with filled circles). Two different cluster models are shown. The $r_{0}=0.21 \mathrm{pc}$ model provides the best fit to the overall cluster, although is not well matched to the central bins. The $r_{0}=$ $0.16 \mathrm{pc}$ model more accurately describes the central cluster.

would indicate adequate agreement of the models with observations. On the other hand, the $\chi^{2}$ results may indicate that the ONC cannot be represented precisely by a simple King model. Because this fitting is scaled to the overall cluster structure, it weights the outer regions where most of the stars reside, and the corresponding central density values are less certain. The best estimates for core properties should probably be derived without the constraint of fitting large-scale cluster structure. Weighting the inner source counts more heavily suggests a smaller core radius and hence a considerably higher central density than that found from fitting the overall cluster with a larger core radius.

The relatively small core radius of the ONC suggests a high concentration value, $c=\log \left(r_{t} / r_{0}\right)$, as well as a large uncertainty in the concentration. Although the limited survey area does not allow us to constrain the truncation radius, $r_{t}$, very well, the fitted model is for $\Psi(0) / \sigma^{2}=9$ or $c \approx 2$ (Binney \& Tremaine 1987, Fig. 4-10), comparable to the concentrations of globular clusters. Given the observational uncertainties, it is somewhat remarkable that a simple, single-mass, velocity-isotropic cluster model fits the ONC data so well. Although the model is unlikely to be correct in detail given that it does not include physical effects known to be present in the ONC, such as the mass spectrum and the potential of the molecular gas, it is difficult to justify more complex modeling at this time. We thus adopt the single-mass, $r_{0}=0.16-0.21 \mathrm{pc}$ King model as a reasonable description of the cluster.

\subsection{Results for Physical Parameters}

We may use the King model comparison in two ways. First, we can use the model as a fitting function to derive intrinsic volume densities of stars from the observed surface densities. This is independent of the appropriateness of the physical assumptions used to construct the model and can be justified by appealing to the comparison in Figure 5 of a spherically symmetric model with the observed surface densities. Second, we may use the model to explore physical conditions in the cluster by assuming that the model is physically correct, and then deriving additional physical quantities to explore possible consistency.

In the limit of high central concentration (which is appropriate for practical purposes here), the relation between the 
TABLE 2

ONC/Trapezium Cluster Parameters

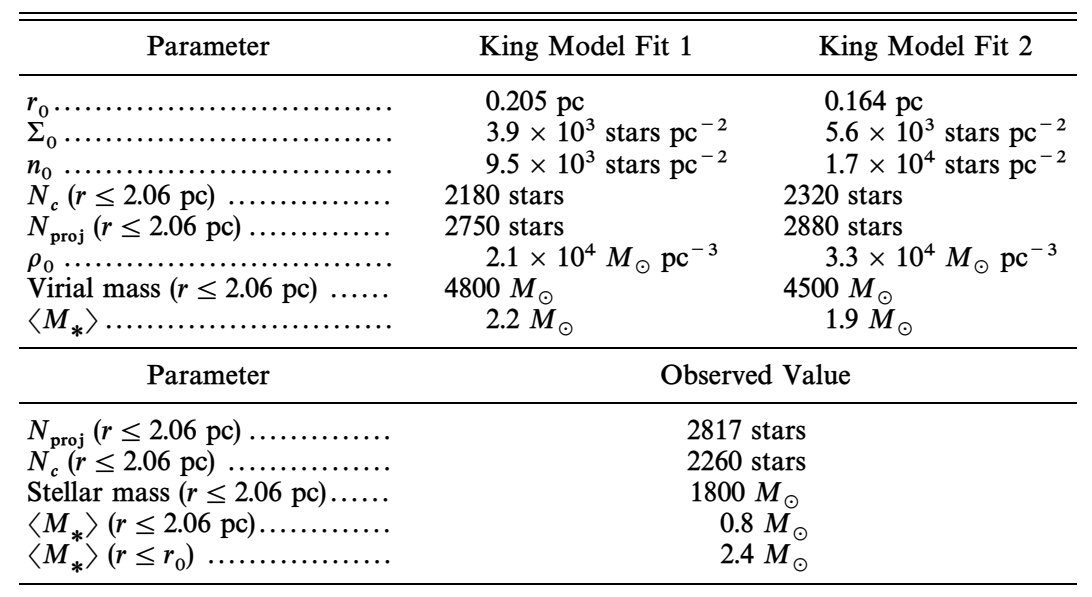

Note. $-N_{c}(r)$ is the true number of stars within radius $r$ of the center; $N_{\text {proj }}(r)$ is the number of stars observed projected on the sky within impact parameter $r$.

central surface density $\Sigma_{0}$ and the true central volume density $n_{0}$ is ${ }^{6}$

$$
\Sigma_{0}=2 n_{0} r_{0} .
$$

From the above discussion we estimate that the core radius lies in the range $r_{0} \approx 0.16-0.21 \mathrm{pc}$, depending on which fit is used. We carry through calculations for both limits, though, as mentioned above, because of the weighting of the outer regions, the larger core radius value does not correspond well to the observed number counts in the center. As shown in Table 2, the best estimate of the central stellar density is $1.7 \times 10^{4} \mathrm{stars}_{\mathrm{pc}}^{-3}$. This value is approximately $40 \%$ of the central density estimated by McCaughrean \& Stauffer from their sample; most of this difference is due to the smaller core radius assumed by McCaughrean \& Stauffer. We note that although the central density and the concentration of the ONC are both comparable to the values found for globular clusters (Djorgovski 1993), the total mass of the ONC is far less than that typical of globular clusters; see discussion below and also Figure 1 of Herbig (1994).

We can also use the model to correct for the presence of stars appearing at smaller projected distances than their true distances. The utility of this exercise extends beyond the current investigation of cluster structure, as we may use the same correction factors from two- to three-dimensional quantities to investigate true (as opposed to projected) radial gradients in the properties of the stellar population (e.g., the fraction of stars with circumstellar disks with cluster radius). We limit our analysis to radii $r \leq 2.06 \mathrm{pc}$ in order to be comfortably inside the region surveyed. This overlaps slightly with the OMC-2 infrared cluster about $14^{\prime}$ north of the ONC core (Fig. 1 [bottom]; see also Ali \& DePoy), but there does not seem to be a large effect from this region. The models suggest that about $20 \%$ of the stars projected within this radius are actually at larger radial distances $^{7}$ from $\theta^{1}$ Ori $\mathrm{C}$; this uncertainty in the outer structure does not strongly affect our estimates of the total

\footnotetext{
${ }^{6}$ Numerical integration of our $c=2$ models yields a coefficient of 1.98, close to the value of 2.00 from the high-concentration limit.

${ }^{7}$ See Table 4-1 of Binney \& Tremaine (1987) for general relationships between true and projected densities for the isothermal sphere.
}

number of stars within this radius. We adopt a limiting radius to the cluster of $2.06 \mathrm{pc}$ in what follows.

Thus far, all conclusions we have drawn are based solely on the observed source counts with radius and the assumption of spherical symmetry. We next investigate the mass needed if the assumption of virial equilibrium is correct, which can be determined from the cluster parameters already in hand and an assumed velocity dispersion. As discussed above, Jones \& Walker found that the averaged radial and tangential velocity dispersions were roughly equal at $\sigma \approx 2.3 \mathrm{~km} \mathrm{~s}^{-1}$, and we use that value here. Table 2 illustrates the results of the following discussion assuming the two values of $\Sigma_{0}$ and $r_{0}$ discussed above.

In the King models the central mass density is related to the one-dimensional (isotropic) velocity dispersion $\sigma$ and the core radius through the relation

$$
\rho_{0}=\frac{9 \sigma^{2}}{4 \pi G r_{0}^{2}} .
$$

The virial mass implied by the model fits and the adopted velocity dispersion for $r \leq 2.06 \mathrm{pc}$ is then $\sim 4500 M_{\odot}$. We may compare this with estimates of the mass in stars in this region. The observed number of stars within this radius is $N_{\text {proj }}=2817$, and we correct this for projection effects to estimate the true number of stars within the appropriate radius as $N_{c} \approx 2260$. An average mass of $\sim 0.8 M_{\odot}$ was observed for the representative sample discussed by Hillenbrand (1997), comparable to the average mass of $\sim 0.7 M_{\odot}$ for the Miller-Scalo (1979) initial mass function (IMF) truncated at $M=0.1 M_{\odot} \cdot{ }^{8}$ The total mass in stars is then $\sim 1800 M_{\odot}$, or about $40 \%$ of the mass required for virial equilibrium. One might worry that this is an inappropriate way to estimate the total cluster mass if sources are still missing from the photometric surveys. Another estimate of the cluster mass may be calculated by assuming that the mass distribution follows the Miller \& Scalo (1979) function, for which $8.5 \%$ of the mass is found in stars more massive than $10 M_{\odot}$. The observed mass of supra-10 $M_{\odot}$

\footnotetext{
${ }^{8}$ Note that while the median mass for this distribution is $0.3 M_{\odot}$, the average mass is $0.7 M_{\odot}$.
} 
stars in the $\mathrm{ONC}$ is $138 M_{\odot}$ for the optically visible sample and $\gtrsim 40 M_{\odot}$ for the infrared sample (counting only BN and IRc2), yielding a predicted total cluster mass of $\sim 2100$ $M_{\odot}$, which is consistent with but higher than the value of $\sim 1800 M_{\odot}$ derived from star counts. Note that these total mass estimates do not include any additional mass contained in the fainter components of unresolved multiple systems. Viewing the issue of cluster equilibrium in an alternative but equivalent manner, the mean mass per star interior to radius $r$ is

$$
\left\langle M_{*}(r)\right\rangle=\frac{\int_{0}^{r} \rho(r) 4 \pi r^{2} d r}{\int_{0}^{r} n(r) 4 \pi r^{2} d r} .
$$

Since the simple model does not include a mass distribution and therefore mass segregation, the mean mass per star is implicitly constant with radius and can be evaluated at the center:

$$
\left\langle M_{*}\right\rangle=\frac{\rho_{0}}{n_{0}} .
$$

Virial equilibrium due to the mass in stars alone would require an average stellar mass of $1.9 M_{\odot}$. This is more than a factor of 2 larger than the observed average mass of $\sim 0.8$ $M_{\odot}$, again indicating that the mass in stars is insufficient for equilibrium. We note, however, that the mass in stars does not fall far below the $\geq 50 \%$ of virial equilibrium that would be required for a negative total energy, i.e., a bound system. We return to this point in $\S 6.3$, where we consider the possible gravitating effects of the gas in the region.

\section{DISCUSSION}

We have used an equilibrium spherical cluster model with stars possessing a Maxwellian velocity distribution to help interpret the geometrical distribution and physical state of the ONC. The structure predicted by this model is not vastly different from that which is observed from stellar source counts. At first thought, it is somewhat surprising that the data look so much like the virial equilibrium, Maxwellian velocity distribution King models. The usual physical interpretation of these models is that the stellar system has relaxed via two-body interactions, thereby achieved energy equipartition, and subsequently undergone mass segregation. In the ONC, however, the cluster is so young that it probably has not gravitationally relaxed, though it may be in the process of virialization via violent relaxation (Binney \& Tremaine 1987). Yet there is also evidence for at least some mass segregation. The question then arises, is the King model comparison physically meaningful? In this section we attempt to address these issues.

\subsection{Relaxation}

Several observational results suggest that the ONC cluster may be so young that it has not collisionally relaxed. These observational indications follow:

1. Cluster elongation.-The ONC clearly is not spherically symmetric, especially on large $(r>0.5 \mathrm{pc})$ scales. Unless the cluster has a large angular momentum, which seems unlikely, this elongation suggests that it has not adjusted from its initial state, especially given the good match between the stellar extension and the molecular gas filamentary distribution in the region.
2. Extreme stellar youth.-The stellar age distribution found in Hillenbrand (1997) indicates that most of the stars have ages less than $1 \mathrm{Myr}$, with the mean age $\sim 0.8 \mathrm{Myr}$. These ages are shorter than the cluster crossing time, $\tau_{c}=$ $2 r / \sigma=2(2.06 \mathrm{pc}) / 2.34 \mathrm{~km} \mathrm{~s}^{-1} \approx 1.8 \mathrm{Myr}$; this number is a lower limit if the cluster is in fact larger than our assumed outer radius of $2.06 \mathrm{pc}$. More than one crossing time is needed for collisional relaxation (see below).

3. Existence of remnant molecular gas.-There is a substantial amount of molecular gas behind the ONC, with sufficient mass (several $\times 10^{3} M_{\odot}$; see $\S 6.3$ ) to have a significant gravitational effect. Unless this material is far in the background of the ONC, which seems unlikely given the partially embedded nature of the cluster, and given that models for the ionization of the Orion Nebula place at least some of this material within about $0.2 \mathrm{pc}$ of $\theta^{1}$ Ori $\mathrm{C}$ (see $\S 6.3$ ), the presence of molecular gas suggests the extreme youth of the associated stellar population. Furthermore, large amounts of remnant gas suggest that some dynamical evolution may be occurring as the central OB stars help disperse this material.

In summary, it appears unlikely from a qualitative standpoint that the cluster has evolved enough from its initial state to have undergone collisional relaxation.

In order to investigate the possibility of cluster relaxation more quantitatively, we first consider the collisional relaxation time that refers to a star of average mass. The halfmass relaxation time is (Spitzer \& Hart 1971; Spitzer 1987)

$$
\tau_{r_{h}}=2 \times 10^{6} \frac{\left(r_{h} / \mathrm{pc}\right)^{3 / 2} N^{1 / 2}}{\left(M_{*} / M_{\odot}\right)^{1 / 2} \ln 0.4 N} \mathrm{yr},
$$

where $r_{h}$ is the half-mass radius of a cluster, $N$ is the number of stars within that radius, and $M_{*}$ is the mean mass per star.

Using our outer region of $2.06 \mathrm{pc}$, the model comparisons ${ }^{9}$ suggest that half of the cluster mass is contained within $r_{h} \sim 0.8 \mathrm{pc}$, for a total number of stars of $N \sim 2800$ (Table 2) and an assumed mean mass per star of $M_{*}=0.7 M_{\odot}$ from the Miller-Scalo IMF. With these values, $\tau_{r_{h}} \sim 6.5 \mathrm{Myr}$, much longer than the H-R diagram ages of the stars, and thus we conclude that the ONC has not had sufficient time for encounters between most stars to decrease any initial departures from a Maxwellian velocity distribution significantly. This long timescale suggests that violent relaxation (Bonnell \& Davies 1997), which may be in the process of occurring, could be partly responsible for the relative mass independence of the velocity dispersion $(\S 4)$. Violent relaxation, which requires a time-varying overall gravitational potential (Binney \& Tremaine 1987), is especially plausible if the molecular gas in the region contributes appreciably to the cluster potential (§ 6.3), since the gas is likely to be evolving rapidly_-perhaps still falling in. On the other hand, the cluster simply could have formed in a nearequilibrium manner.

Although the ONC as whole has not yet collisionally relaxed, the inner core (i.e., the Trapezium region) may have. Applying the more general collisional relaxation time estimate (Lightman \& Shapiro 1977; Spitzer 1987) to the

\footnotetext{
9 The half-mass radius produced by the model, $0.8 \mathrm{pc}$, agrees with the value of $0.8 \mathrm{pc}$ calculated from the optical analysis for the projected radius within which $\frac{1}{2}$ of the optically visible mass is contained.
} 
innermost regions,

$$
\tau_{r}=3.4 \times 10^{9} \frac{\left(\sqrt{3} \sigma / \mathrm{km} \mathrm{s}^{-1}\right)^{3}}{\left(n / \mathrm{pc}^{-3}\right)\left(M_{*} / M_{\odot}\right)^{2} \ln 0.4 N} \mathrm{yr} .
$$

Adopting the core parameters (and including projection effects) $n_{0}=1.7 \times 10^{4} \mathrm{pc}^{-3}, N_{0} \sim 2.1 n_{0} r_{0}^{3} \sim 160$ stars, $\sigma_{0}=2.5 \mathrm{~km} \mathrm{~s}^{-1}$, and $\left\langle M_{*}\right\rangle_{0}=2.4 M_{\odot}$, we find $\tau_{r}(r=0) \sim$ $0.6 \mathrm{Myr}$, comparable to the mean stellar age in the inner cluster.

\subsection{Mass Segregation}

The existence of a mass spectrum in the ONC implies that the true dynamical situation is more complicated than our initial simplified treatment. The most significant difference between the single-mass and the multimass models (for our discussion of the ONC) is that the multimass model evolves on a shorter timescale than the single-mass model does. If the system is older than a dynamical time but less than a collisional relaxation time, encounters between stars are important and massive stars can impart energy to less massive stars, leading to more rapid mass segregation than suggested by equations (5) and (6). In this case, the core radius will be different for different mass components, $m_{i}$. These individual mass components will evolve relative to the cluster as a whole on timescales proportional to $\langle M\rangle / m_{i}$ (Spitzer 1987; see also Chernoff \& Weinberg 1990).

There is indeed clear evidence for mass segregation in the ONC, with the highest mass stars preferentially found toward the center of the cluster as has been known for over five decades. To test whether there is evidence for more general mass segregation, we present in Figure 6 cumulative distributions with radius of stars contained within different mass intervals. This figure includes only the 665 stars currently located on the theoretical H-R diagram that are also designated as proper-motion cluster members (note that this requirement removes many likely cluster members that simply do not have measured proper motions). We show four different panels to illustrate the sensitivity of the cumulative plots to the limiting radius. Inside $1.0 \mathrm{pc}$, "general" mass segregation appears to be established in the ONC, with stars of masses less than 0.3, 0.3-1.0, 1.0-5.0, and greater than $5 M_{\odot}$ progressively more centrally concentrated with increasing mass. Beyond $1.0 \mathrm{pc}$, the evidence for general mass segregation is less compelling than at smaller projected radii. Two-sample Kolmogorov-Smirnov tests confirm the statement that the radial distribution of the highest mass stars is distinguished from all others at very high confidence levels, $P<10^{-3}$. Even out to the edge of the area surveyed, the greater than $5 M_{\odot}$ component shows clear central concentration. The cumulative radial distributions for the lower mass bins cannot, however, be distinguished from one another to satisfactory statistical significance at all cluster radii. Experimentation with the mass bins leads us to conclude that there is some evidence for general mass segregation down to $\sim 1-2 M_{\odot}$, but not below this.

We can also quantify the mass segregation by investigating the mean stellar mass as a function of projected cluster radius. Averaged over all radii $r<2.5 \mathrm{pc}$, the mean stellar mass derived from the optical H-R diagram is $0.72-0.85$ $M_{\odot}$ (depending on which set of pre-main-sequence tracks is used), similar to the Miller \& Scalo value of $0.7 M_{\odot}$. As a function of projected radius, the mean stellar mass appears

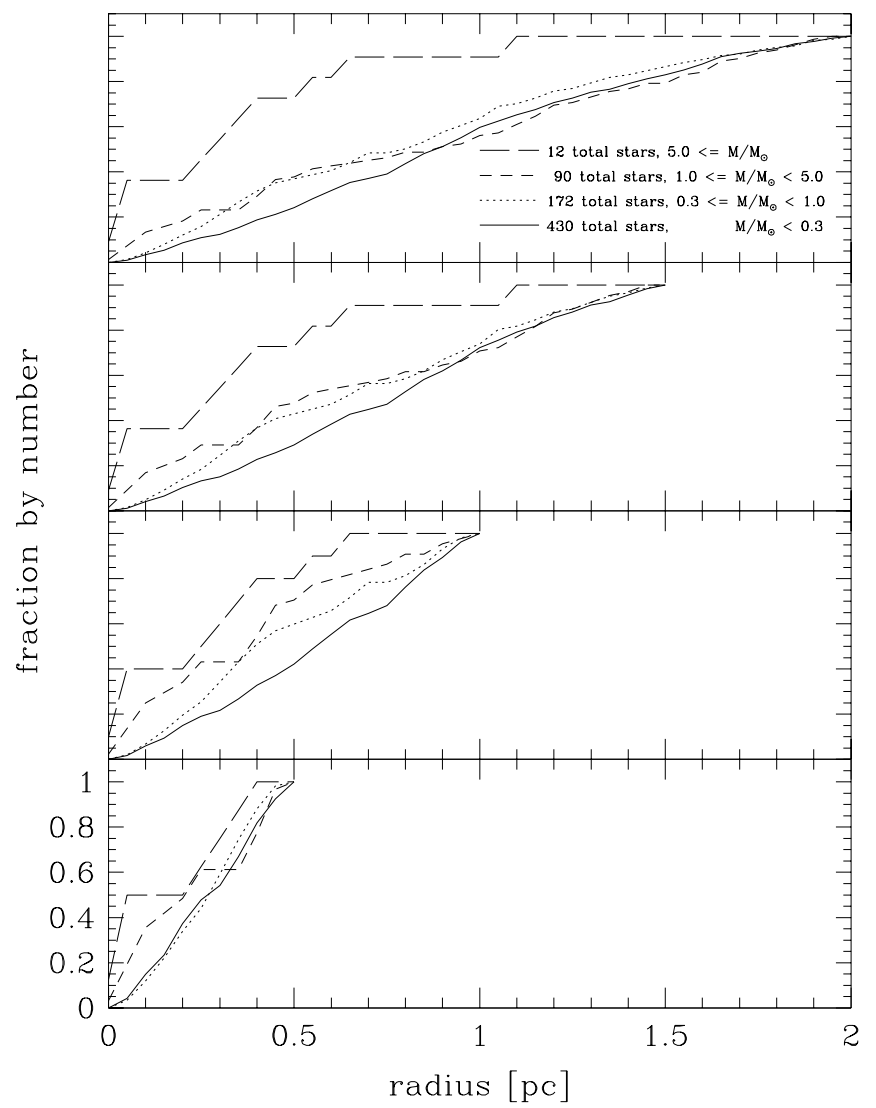

Fig. 6.-Cumulative radial distributions of source counts over different mass intervals. Four different limiting radii are shown in the four panels, to illustrate the sensitivity of the cumulative plots to the outer radius. This figure shows clear evidence for segregation of stars more massive than $5 M_{\odot}$ (long-dashed lines) toward the cluster center and some evidence for general mass segregation persisting down to $1-2 M_{\odot}$.

relatively flat within the errors inward to less than $0.1 \mathrm{pc}$ $\left(\sim \frac{1}{2} r_{0}\right)$, where it then begins to rise toward smaller radii. This effect is not due to incompleteness of the spectroscopic sample since it remains the case even when all optical stars without mass estimates (i.e., spectral types) are assumed to have an average mass. We interpret the increase in the mean mass per star with decreasing radius as additional evidence for segregation of the most massive stars toward the inner cluster. Within $0.05 \mathrm{pc}$ the mean mass per star rises above $\sim 2-4 M_{\odot}$ to a peak value of $\sim 6-9 M_{\odot}$ (where the range is derived from assuming average masses for all optical stars without mass estimates vs. including only those stars with known spectral types). If $\theta^{1}$ Ori $\mathrm{C}\left(M \approx 50 M_{\odot}\right)$ is not counted in these calculations, the mean mass rises to a peak value of just $\sim 1.3-2 M_{\odot}$, but still twice the mean mass at all radii, $r>0.05 \mathrm{pc}$.

Is the observed mass segregation a primordial feature of the cluster or the result of dynamical evolution? Standard calculations (Spitzer \& Shull 1975) suggest that dynamical mass segregation occurs on a timescale $\sim \tau_{r_{h}}$, although the appropriateness of $\tau_{r h}$ for anything but the mean stellar mass (eq. [5]) should be clarified. According to Spitzer (1987), the relative timescale for the dynamical evolution of two different mass components is

$$
\frac{\tau_{1}}{\tau_{2}} \approx 0.44 \frac{m_{2}}{m_{1}}\left(1+\frac{\sigma_{1}^{2}}{\sigma_{2}^{2}}\right)^{3 / 2} .
$$


Setting $\tau_{2}=\tau_{r_{h}}, m_{2}=\left\langle M_{*}\right\rangle=0.8 M_{\odot}$, and $\sigma_{2}=2.34 \mathrm{~km}$ $\mathrm{s}^{-1}$, we find that $10 M_{\odot}$ stars with a velocity dispersion of $1.5 \mathrm{~km} \mathrm{~s}^{-1}$ (§ 4) should evolve in $\sim 6 \%$ of the time required for evolution of a star of average mass and average velocity dispersion. With $\tau_{r_{h}}=6.5 \mathrm{Myr}$ and a cluster age of less than $1 \mathrm{Myr}$ but greater than $0.06 \times 6.5 \mathrm{Myr} \gtrsim 0.4 \mathrm{Myr}$, these results suggest that the positions of the most massive stars may be due, in part, to dynamical evolution. More sophisticated numerical modeling of clusters similar to the ONC in terms of the total number of stars and the mass spectrum by Bonnell \& Davies (1997) show that some degree of mass segregation (but not full mass segregation) does indeed occur within just a few crossing times. The timescale for segregation depends most strongly on the number of stars in the cluster and on the relationship between the velocity dispersion and stellar mass [with clusters containing smaller numbers of stars and steeper $\sigma(M)$ relationships segregating more quickly]. Their simulations also include clusters (1) in and out of virial equilibrium, (2) of spherical and oblate shape, and (3) of different radial surface density profiles; little dependence on any these variables is found. Bonnell \& Davies (1997) claim that in order to produce a Trapezium-type system (three of the six most massive stars located in the inner $0.1 r_{h}$ ) within just a few crossing times, these massive stars most likely formed within the inner $10 \%$ of the cluster. Like these authors, we conclude based our analysis above that the structure of the ONC is more likely the result of initial conditions than the result of dynamical evolution. The morphology of the Trapezium $\mathrm{H}$ II region also supports a picture in which its ionizing $\mathrm{O}$ and $\mathrm{B}$ stars probably did not form far from where they are located today - at its center and at the center of the extended stellar cluster.

Such a "core-halo" structure, in which higher mass stars are concentrated toward the centers of extended lower mass clusters, has been found previously for other relatively young stellar systems. These include IC $1805(\tau=1-3 \mathrm{Myr}$; Sagar et al. 1988); NGC 6530 ( $\tau=1-3 \mathrm{Myr}$; McNamara \& Sekiguchi 1986); NGC $6913(\tau=4-6 \mathrm{Myr}$; Sagar et al. 1988); NGC 3293 ( $\tau=6-23$ Myr; Herbst \& Miller 1982); NGC $654(\tau=17 \mathrm{Myr}$; Stone 1980); NGC $581(\tau=3-30$ Myr; Sagar et al. 1988); $\mathrm{h}$ and $\chi \operatorname{Per}(\tau=20-30 \mathrm{Myr}$ and 10-27 Myr, respectively; Vogt 1971); $\operatorname{Tr} 1(\tau=25 \mathrm{Myr}$; Sagar et al. 1988); M35 $(\tau=20-40 \mathrm{Myr}$; Mathieu 1983a, $1983 \mathrm{~b})$; and the Pleiades ( $\tau=70-120 \mathrm{Myr}$; van Leeuwen 1983). In all of these cases, the ages are within a factor of about $2-3$ of the average collisional relaxation times, and so given errors of the same magnitude in the relaxation time estimates, the evidence for primordial mass segregation is generally weak. However, "core-halo" structure is also found for the young embedded clusters Mon R2 (Carpenter et al. 1997) and NGC 2024 (Meyer et al. 1998) where, like the ONC, the total potential still has a significant contribution from a molecular gas component that is usually thought to impede the process of dynamical mass segregation. That the outer regions of the ONC and of the embedded clusters are not even 1 crossing time old argues strongly that any mass segregation must be primordial. By analogy, we suggest that at least some of the core-halo appearance of the other young clusters mentioned above may be a product of the star formation process itself and not caused solely via dynamical evolution. This suggestion is similar to the conclusions on the nature of mass segregation in relatively young stellar systems reached by Sagar et al. (1988) and by
McNamara \& Sekiguchi (1986). Larson (1982) conjectured similarly, based on morphological evidence alone, that massive stars must form in dense cluster centers.

\subsection{Boundedness}

As discussed in $\S 5.2$, the comparison between models and the observed stellar population suggests that the stellar mass in the ONC is $\sim 40 \%$ of that needed for virial equilibrium. However, before concluding that the ONC is not close to virial equilibrium, we need to consider the gravitational potential of the gas in the region.

How much mass in gas is there? The filament of dense molecular gas out of which the ONC was recently formed extends in a north-south direction: $\sim 5 \mathrm{pc}$ to the north of and $\sim 3$ pc to the south (before bending eastward and fanning out into the generally lower density L1641 cloud) of $\theta^{1}$ Ori C, according to the maps of Bally et al. (1987). Although the molecular surveys $\left({ }^{13} \mathrm{CO}\right.$ by Bally et al. 1987 ; $\mathrm{C}^{18} \mathrm{O}$ by Dutrey et al. 1993; CS by Tatematsu et al. 1993; and $\mathrm{NH}_{3}$ by Cesaroni \& Wilson 1994) cover just about all of the $\sim 4.1 \times 4.6 \mathrm{pc}$ area considered here, most of the gas (as well as most of the stellar population) is concentrated in an area just $\sim 1 \mathrm{pc}$ wide.

Goldsmith, Bergin, \& Lis (1997) find $580 M_{\odot}$ contained within $\sim 0.36 \times 1.4 \mathrm{pc}$, while Bally et al. (1987) find 2200 $M_{\odot}$ within $\sim 1 \times 2.1 \mathrm{pc}$, and Castets et al. (1990) find about $8000 M_{\odot}$ over an $8 \mathrm{pc}$ north-south extension. Although the techniques used in these studies to estimate the gas masses are quite different, we are encouraged that the masses do appear to correlate directly with the length of the filament over which measurements were made. If, therefore, we can safely scale from these estimates to the $4.1 \mathrm{pc}$ length considered in this paper for the stellar population, we estimate about $4000 M_{\odot}$ in gas. Suggestively, this is comparable to the virial mass derived from the cluster model and about twice the mass known to exist in stars.

The precise gravitating effect of this molecular gas is somewhat uncertain. It clearly lies behind the Orion Nebula (see the review in Wen \& O'Dell 1995), but its distance behind the stellar cluster is unknown. If the depth of the molecular cloud is comparable to its width on the sky, it cannot be much deeper than 1 pc. Since models for the ionization of the nebula indicate that the distance from $\theta^{1}$ Ori C to the ionization front is only $0.2 \mathrm{pc}$ (Wen \& O'Dell 1995), this strongly suggests that the molecular gas must be just immediately behind the optical stars and, thus, that it contributes strongly to the gravitational potential in the region. Several factors that suggest a close relationship between the molecular material and the current stellar cluster - the large mass in molecular gas, its high column density, its coincidence on the sky with the stellar cluster in terms of projected width and north-south elongation, and the fact that the molecular gas reaches to within almost one core radius of the central stars of the Trapezium - point to a picture in which much star formation has yet to occur in the ONC. Indeed, the BN and KL regions lie a projected distance on the sky comparable to the core radius as well, and the associated cluster of radio sources (Menten \& Reid 1995) may represent just-forming stars that will eventually merge with the rest of the ONC.

Since the mass in stars is already $\sim 40 \%$ of that required for virial equilibrium, if even a modest fraction $(10 \%-20 \%)$ of the molecular gas remaining in the region forms stars, it appears likely that the $\mathrm{ONC}$ will be ultimately bound gravi- 
tationally. There may yet be some time for this star formation to occur. The Orion Nebula is thought to be a "blister" H II region, in which an ionization front moves into a molecular cloud and the ionized gas flows away from the front at nearly the sound speed of $\sim 10 \mathrm{~km} \mathrm{~s}^{-1}$ (see Baldwin et al. 1991 and references therein). Wen \& O'Dell (1995) estimate that the ionization front may be moving into the cloud (if $\theta^{1}$ Ori $\mathrm{C}$ is not moving) at about $0.5 \mathrm{~km}$ $\mathrm{s}^{-1}$, which would suggest that it might take $2 \mathrm{Myr}$ for the front to pass through the molecular gas. Alternatively, we might estimate the lifetime of the molecular gas from the evaporation timescale. Directly behind $\theta^{1}$ Ori $\mathrm{C}$ the electron density in the region just above the ionization front is $\sim 5 \times 10^{3} \mathrm{~cm}^{-3}$ (Wen \& O'Dell 1995); allowing this material to expand at $10 \mathrm{~km} \mathrm{~s}^{-1}$ results in an estimated particle loss of $\sim 5 \times 10^{9} \mathrm{~cm}^{-2} \mathrm{~s}^{-1}$. If the reservoir of molecular material has an $A_{V} \sim 50$, or a column density $\sim 10^{23} \mathrm{~cm}^{-2}$, then the evaporation timescale is $0.6 \mathrm{Myr}$, again comparable to the estimated ages of stars in the region.

Wen \& O'Dell (1995) emphasize the importance of the motion of $\theta^{1}$ Ori $\mathrm{C}$ (which is poorly known) in causing changes in the ionization of the molecular gas and the structure of the nebula. However, no matter where $\theta^{1}$ Ori $C$ is moving, or how fast, it cannot plausibly scour out all of the molecular gas on a timescale much less than $1 \mathrm{Myr}$, since it can only move in one direction. In this connection it is important to remember that most of the mass of the ONC and the associated gas lies on scales $\sim 1-2 \mathrm{pc}-$ much larger than the core. The observational situation suggests the "turning-on" of the massive stars may not stop low-mass star formation instantaneously; the timescale for disruption of the very dense molecular gas after massive star formation may be a significant fraction of the overall duration of cluster formation.

\section{CONCLUSIONS}

Based on comparison of current optical and infrared stellar surveys of the ONC with simple virial equilibrium cluster models, we have argued that the Trapezium "cluster" is most naturally viewed not as a distinct entity but rather as the core region of the larger ONC. From this point of view it also seems plausible that the $\mathrm{BN}$ and $\mathrm{KL}$ regions, which probably lie within $0.5 \mathrm{pc}$ of the ONC center, may be part of the same cluster and represent the end phases of rapid star formation in this remarkably young and dense region.

Several lines of evidence suggest that the stellar distribution has not collisionally relaxed on large scales. The elongation of the cluster directly traces the general north-south filamentary distribution of molecular gas in the region and probably represents the initial conditions of cluster forma- tion by gravitational fragmentation from filamentary molecular gas. Also, the stellar ages are lower by factors of several than the standard timescales needed for collisional relaxation.

Stars of higher mass in the ONC are preferentially found closer to the cluster center than stars of lower mass. This appears not to be the result of dynamical mass segregation, since several collisional relaxation times are needed for mass segregation. These results provide limited observational support for the idea that the formation of higher mass stars can be enhanced by environmental conditions. The high gas densities that must have been present in the ONC core could have provided an ideal environment for the rapid accretion thought to be necessary to form highmass stars (e.g., Bonnell et al. 1997).

The likelihood that the ONC is not collisionally relaxed even though it shows mass segregation makes it perhaps surprising that the King models are a reasonable representation of the (azimuthally averaged) density structure. According to Binney \& Tremaine (1987) and to the simulations of Bonnell \& Davies (1997), $\sim 10$ crossing times are needed to approach virial equilibrium. Not even the centermost portions of the ONC, where the crossing (and thus two-body relaxation) times are the shortest, are that old. If, however, violent relaxation is important, the stars could achieve an approximately mass-independent Maxwellian velocity distribution within just one or a few dynamical times, and so the cluster might not need to undergo standard two-body relaxation before appearing to resemble a standard virialized cluster model. On the other hand, the cluster simply could have formed nearly in virial equilibrium.

We estimate that the current mass in stars is within a factor of 2 of that needed for virial equilibrium, suggesting that at least some portion of the ONC may be gravitationally bound. If even a modest fraction of the remnant molecular gas in the region will eventually form stars, it seems likely that in a few Myr the ONC will become a gravitationally bound open cluster.

We have benefited from useful discussions on cluster dynamics with C. Murali and I. Bonnell and on the ONC in general with J. Stauffer. We also thank the referee, Douglas Heggie, for his careful review of our manuscript. Support to L. A. H. for this work was provided by NASA through grant HF1060.01-94A from the Space Telescope Science Institute, which is operated by the Association of Universities for Research in Astronomy, Inc., under NASA contract NAS5-26555. L. W. H. was supported in part by NASA grant NAGW-4282. Publication of this work was supported in part by a grant to L. A. H. from NASA administered by the American Astronomical Society.

\section{REFERENCES}

Ali, B., \& DePoy, D. 1995, AJ, 109, 709

Andrews, A. D. 1981, A Photometric Atlas of the Orion Nebula (Armagh: Armagh Obs. Press)

Baade, W., \& Minkowski, R. 1937, ApJ, 86, 199

Baldwin, J. A., Ferland, G. J., Martin, P. G., Corbin, M. R., Cota, S. A., Peterson, B. M., \& Slettebak, A. 1991, ApJ, 374, 580

Bally, J., Langer, W. D., Stark, A. A., \& Wilson, R. W. 1987, ApJ, 312, L45

Bergin, E. A. 1996, Ph.D. thesis, Univ. Massachusetts

Binney, J., \& Tremaine, S. 1987, Galactic Dynamics (Princeton: Princeton Univ. Press)

Bonnell, I. A., Bate, M. R., Clarke, C. J., \& Pringle, J. E. 1997, MNRAS, 285,201

Bonnell, I. A., \& Davies, M. B. 1997, MNRAS, submitted
Carpenter, J. M., Meyer, M. R., Dougados, C., Strom, S. E., \& Hillenbrand, L. A. 1997, AJ, 114, 198

Castets, A., Duvert, G., Dutrey, A., Bally, J., Langer, W. D., \& Wilson, R. W. 1990, A\&A, 234, 469

Cesaroni, R., \& Wilson, T. L. 1994, A\&A, 281, 209

Chernoff, D. F., \& Weinberg, M. D. 1990, ApJ, 351, 12

Djorgovski, S. 1993, in Structure and Dynamics of Globular Clusters, ed. S. G. Djorgovski \& G. Meylan (ASP: San Francisco), 325

Dutrey, A., Duvert, G., Castets, A., Langer, W. D., Bally, J., \& Wilson, R. W. 1993, A\&A, 270, 468

Fallon, F. W., Gerola, H., \& Sofia, S. 1977, ApJ, 217, 719

Goldsmith, P. F., Bergin, E. A., \& Lis, D. C. 1997, ApJ, 491, 615

Herbig, G. H. 1994, Rev. Mexicana Astron. Astrofis., 29, 17 
Herbig, G. H., \& Terndrup, D. M. 1986, ApJ, 307, 609

Herbst, W., \& Miller, D. P. 1982, AJ, 87, 1478

Hillenbrand, L. A. 1997, AJ, 113, 1733

Hillenbrand, L. A., Strom, S. E., Makidon, R. B., Merrill, K. M., Gatley, I., Calvet, N., Skrutskie, M. F., \& Meyer, M. R. 1998, submitted

Johnson, H. L. 1965, ApJ, 142, 964

Jones, B. F., \& Walker, M. F. 1988, AJ, 95, 1755

Larson, R. B. 1982, MNRAS, 200, 159

Lightman, A. P., \& Shapiro, S. L. 1977, ApJ, 211, 244

Mathieu, R. D. 1983a, ApJ, 267, L97

.1983b, Ph.D. diss., Univ. California, Berkeley

McCaughrean, M. J. 1987, Ph.D. thesis, Edinburgh Univ.

McCaughrean, M. J., \& Stauffer, J. 1994, AJ, 108, 1382

McCaughrean, M. J., Zinnecker, H., Rayner, J. T., \& Stauffer, J. R. 1995, in The Bottom of the Main Sequence and Beyond, ed. C. Tinney (Berlin: Springer), 209

McNamara, B. J., \& Sekiguchi, K. 1986, ApJ, 310, 613

Menten, K. M., \& Reid, M. J. 1995, ApJ, 445, L157

Meyer, M. R. 1996, Ph.D. thesis, Univ. Massachusetts

Meyer, M. R., Carpenter, J. M., Hillenbrand, L. A., \& Strom, S. E. 1998, in preparation

Miller, G. E., \& Scalo, J. M. 1979, ApJS, 41, 513

O’Dell, C. R., \& Wong, S. K. 1996, AJ, 111, 846
Parenago, P. P. 1954, Trudy Sternberg Astron. Inst., Vol. 25

Prosser, C. F. F., Stauffer, J. R., Hartmann, L. W., Soderblom, D. R., Jones, B. F., Werner, M. W., \& McCaughrean, M. J. 1994, ApJ, 421, 517

Retterer, J. M. 1979, AJ, 84, 370

Sagar, R., Miakutin, V. I., Piskunov, A. E., \& Dluzhnevskaia, O. B. 1988, MNRAS, 234, 831

Spitzer, L. 1987, Dynamical Evolution of Globular Clusters (Princeton: Princeton Univ. Press)

Spitzer, L., \& Hart, M. H. 1971, ApJ, 164, 399

Spitzer, L., \& Shull, J. M. 1975, ApJ, 201, 733

Stauffer, J. R., Prosser, C. F., Hartmann, L., \& McCaughrean, M. J. 1994, AJ, 108, 1375

Stone, R. C. 1980, PASP, 92, 426

Tatematsu, K., et al. 1993, ApJ, 404, 643

Trumpler, R. J. 1931, PASP, 43, 255

van Altena, W. F., Lee, J. T., Lee, J. F., Lu, P. K., \& Upgren, A. R. 1988, AJ, 95,1744

van Leeuwen, F. 1983, Ph.D diss., Sterrewacht, Leiden

Walker, M. F. 1969, ApJ, 155, 447

.1983, ApJ, 271, 642

Wen, Z., \& O'Dell, C. R. 1995, ApJ, 438, 784

Vogt, N. 1971, A\&A, 11, 359 